\title{
Metodología para la evaluación de las competencias laborales en salud
}

\section{Methodology for the work performance evaluation in the health sector}

\section{Omayda Urbina Laza}

Doctora en Ciencias de la Salud. Profesor Titular. Escuela Nacional de Salud Pública. La Habana, Cuba.

\section{RESUMEN}

Se describe una metodología para evaluar competencias laborales en profesionales del sector de la salud, diseñada en una investigación del Programa Ramal de Investigación en Sistemas y Servicios de Salud de Cuba, que se realizó entre el 2002 y 2004 y se validó en los Servicios de Neonatología de Ciudad de La Habana, los resultados fueron utilizados para la idoneidad demostrada del personal de enfermería del Ministerio de Salud Pública. El algoritmo de la investigación se estructuró en tres etapas con el uso de técnicas cualitativas, tales como revisión documental, consulta a dos grupos diferentes de expertos: un comité de experto de la especialidad y el segundo conformado por otros profesionales de la salud; la observación en los servicios y la aplicación de una auditoria. Se aplicó una encuesta a los profesionales para conocer aspectos que consideraban como insuficientes en su formación para trabajar en los servicios de salud y las causas identificadas por ellos. El carácter dialéctico y participativo de la metodología propuesta permite que con cada etapa se enriquezca la otra y se logre un producto científicamente probado.

Palabras clave: Competencias, profesionales, metodología, investigación. 


\section{ABSTRACT}

A methodology was presented in detail to evaluate work competencies in the health professionals. It was designed in a research work within the Branch Program of Research on Health Systems and Services in Cuba carried out from 2002 to 2004 and then validated in the Neonatology Services in the City of Havana province. The results were used to determine the suitability of the nursery staff of the Ministry of Public Health. The algorithm of this research work was structured in three phases using qualitative techniques such as documentary review, consultations with two different expert teams, one expert committee of the specialty and the other made up of other health professionals, service observation, and auditing. The professionals were administered a survey to find out those aspects that they considered poor in their formation as health service workers, and the causes. The dialectical and participatory nature of the presented methodology makes possible that one phase enhances the following phase, and thus a scientifically proven product was achieved.

Key words: Competencies, professionals, methodology, research.

\section{INTRODUCCIÓN}

La formación para el trabajo es una mezcla entre educación, experiencia laboral y formación específica adquirida a lo largo de la vida, de ahí que las competencias se definen y se construyen en la práctica social y son una tarea conjunta entre empresas, trabajadores y educadores. ${ }^{1}$

Las competencias son valoradas como una herramienta capaz de proveer una conceptualización, un modo de hacer y un lenguaje común para el desarrollo de los recursos humanos, constituyen una visión y organización sistemática y se han expresado como un sistema de certificación legalmente establecido en varias regiones del mundo, incluida América Latina; se trata de un vínculo entre trabajo, educación y capacitación. ${ }^{2}$

El estudio de las competencias laborales, rebasa la mera significación económicolaboral del término, para proyectarse como componente importante en el desarrollo integral de los profesionales, a partir de la asunción de un aprendizaje donde se acrecienten las capacidades humanas, se garantice un desempeño eficiente y responsable y se propicie el incremento de las posibilidades de realización personal y social del individuo. Desde la perspectiva de los servicios de salud, las competencias profesionales abarcan los aspectos cognoscitivos de la clínica y otros necesarios para el adecuado desempeño de la profesión, entre ellos se encuentran: la relación profesional-paciente y la estrecha relación que debe existir entre asistencia-investigación-docencia y administración.

Una competencia (en el sentido técnico del capital humano organizativo) es un conjunto de atributos que una persona posee y le permiten desarrollar acción efectiva en determinado ámbito. Es la interacción armoniosa de las habilidades, conocimientos, valores, motivaciones, rasgos de personalidad y aptitudes propias 
de cada persona que determinan y predicen el comportamiento que conduce a la consecución de los resultados u objetivos a alcanzar en la organización.

Dentro de las organizaciones, las competencias son utilizadas para potenciar el capital humano en pos de los objetivos del puesto, área y organización y también para desarrollar al ser humano.

En el análisis de las tendencias y enfoques de las competencias, se ha constatado que se le han ido añadiendo diferentes aspectos y enriqueciendo con otros; existen disímiles definiciones, las cuales toman en cuenta el contexto, para quién, por qué y con qué fin. Se observa en los autores estudiados acepciones diferentes del término, unos la nombran como competencia, otras competencias, competencias profesionales y competencias laborales; esta última es la que utiliza la Organización Internacional del Trabajo y la que se asume en este artículo. Se presentan algunas de las más utilizadas:

- La competencia laboral se identifica en las normas a través de la definición de elementos de competencia: criterios de desempeño, campo de aplicación y conocimientos requeridos. ${ }^{3}$

- Las competencias profesionales como el conjunto de capacidades, comportamientos, conocimientos, habilidades y actitudes que permiten el desempeño de una ocupación para alcanzar los resultados pretendidos en un determinado contexto laboral. ${ }^{4-7}$

- La competencia laboral es la construcción social de aprendizajes útiles para el desempeño de una actividad productiva en un determinado contexto laboral, de acuerdo con los requerimientos de calidad esperados por el sector productivo. ${ }^{4-6}$

- Las competencias como la capacidad de movilizar saberes, capacidades socioafectivas, habilidades, actitudes, valores y comportamientos que ocasionan un desempeño exitoso en las funciones y tareas a desarrollar en correspondencia con el principio de la idoneidad demostrada. ${ }^{4-7}$

Todos estos referentes teóricos llevaron a definir las competencias laborales como:

...la integración de conocimientos, habilidades, conductas, actitudes, aptitudes y motivaciones conducentes a un desempeño adecuado y oportuno en diversos contextos; también responde a las funciones y tareas de un profesional para desarrollarse idóneamente en su puesto de trabajo y es el resultado de un proceso relacionado con la experiencia, capacitación y calificación. ${ }^{4}$

Los estudios sobre el tema de las competencias han estado dirigidos a la búsqueda de necesidades de aprendizaje, que constituyen el punto de partida de estrategias de capacitación sobre problemas detectados y establecer un sistema de seguimiento que garantice incentivar la autosuperación individual como vía para lograr el perfeccionamiento técnico y profesional que posibilite la transformación cualitativa de los servicios de salud.

La evaluación de las competencias laborales tiene la peculiaridad de que se lleva a cabo durante el proceso de trabajo y pone de manifiesto la efectividad del desempeño laboral y las deficiencias e insuficiencias de los trabajadores en su actividad profesional cotidiana. En su sentido más amplio es aplicada en las diferentes aristas de la vida profesional, laboral y educacional. Evaluar es emitir un juicio de valor que expresa la magnitud y calidad con que se han logrado los objetivos propuestos. La evaluación es el proceso de identificar, obtener y analizar 
información útil, que permite valorar y enjuiciar los fenómenos que se presentan en torno al objeto, con el fin de obtener criterios y juicios sobre el mismo, para tomar decisiones y solucionar problemas. ${ }^{5}$

La evaluación como proceso, es un instrumento clave para las instituciones, los directivos y los trabajadores, mediante ella se puede articular el potencial de las competencias con una adecuada proyección estratégica para el desarrollo de los profesionales, de ahí que resultan indispensables las respuestas a la pregunta ¿por qué evaluar las competencias laborales? Las respuestas pudieran estar comprendidas en los siguientes aspectos:

- Organizar y movilizar las competencias.

- Desarrollar el potencial de competencias.

- Brindar información relacionada con el desempeño demostrado por los profesionales.

- Integrar y retroalimentar a los procesos de desarrollo y capacitación permanente de los profesionales.

Según Fernando Vargas, estos son los principios que orientan la evaluación de las competencias laborales: ${ }^{6}$

- Validez. Basada en información precisa y suficiente para la emisión de juicios.

- Confiabilidad. Debe producir resultados consistentes en aplicaciones a personas, contextos y momentos diversos.

- Relevante. Objetivos y resultados importantes para los participantes.

- Accesibilidad y equidad. Se debe evitar toda práctica discriminatoria.

- Viabilidad/factibilidad. Un diseño posible en un contexto específico y llevado a cabo por personas capacitadas.

- Flexibilidad. Adaptarse a diferentes modalidades y necesidades.

El gran desafío de los evaluadores es precisamente ¿cómo evaluar las competencias?, ¿qué modelos utilizar, para que estas tengan los principales atributos que la caracterizan? Algunos autores coinciden que se debe realizar mediante el desempeño, pues existe una zona de interacción, entre el "saber cómo se actúa" y el "actuar" que permite retroalimentar al profesional para su actuación competente.

Hasta el momento no existe ningún método, procedimiento, ni técnica ideal para evaluar las competencias laborales, de manera que resulta indispensable vincular todo lo que sea factible y pertinente para lograr una evaluación lo más real y objetiva posible, para ello se consideran necesarios instrumentos diseñados metodológicamente y escenarios que sean afines a las áreas del conocimiento y a los modos de actuación de los profesionales, que verifiquen como se organizan y planifican las acciones a emprender y empleen de forma eficiente el capital humano y financiero. 
El propósito de esta publicación es explicar cada una de las etapas que conforman una metodología, que se propone para la evaluación de las competencias laborales en salud.

\section{METODOLOGÍA PARA LA EVALUACIÓN DE LAS COMPETENCIAS LABORALES EN SALUD}

Se denomina metodología al análisis de los diversos procedimientos concretos que se emplean en las investigaciones y la discusión acerca de sus características, cualidades y debilidades. Otra acepción se refiere a los pasos y procedimientos que se han seguido en una indagación determinada, para designar los modelos concretos de trabajo que se aplican en una determinada disciplina o especialidad. ${ }^{7}$

Se presenta la metodología ECLAS que provee las vías para que la concepción teórica sea aplicable en la práctica e incluye instrumentos y procedimientos validados para su uso, se concibió mediante la construcción del conocimiento que integra componentes teóricos y prácticos. La integración de cada uno de los componentes de la metodología para la evaluación de las competencias laborales y su interacción dinámica le da el carácter sistémico, donde cualquier cambio en uno afectará a los demás.

El enfoque sistémico, significa que el modo de abordar los objetos y fenómenos no puede ser aislado, sino que tiene que verse como parte de un todo. No es la suma de elementos, sino un conjunto de elementos que se encuentran en interacción, de forma integral, que produce nuevas cualidades con características diferentes, cuyo resultado es superior al de los componentes que lo forman y provocan un salto de calidad. ${ }^{8}$

\section{DEFINICIÓN DE LA METODOLOGÍA ECLAS}

Integración de técnicas y procedimientos de la investigación para definir la evaluación (E) de las competencias (C) laborales (LA) en los profesionales de la salud (S).

La metodología ECLAS, presentada en una tesis doctoral, ${ }^{9}$ se diseñó en una investigación del Programa Ramal de Investigación en Sistemas y Servicios de Salud de Cuba, que se realizó entre el 2002 y 2004, se validó en los Servicios de Neonatología de Ciudad de La Habana y los resultados fueron utilizados para la idoneidad demostrada del personal de enfermería del Ministerio de Salud Pública.

\section{Características}

- Es creada a partir de una investigación y para la cual debe constituirse un equipo de trabajo con su investigador principal.

- Responde a las competencias laborales de los profesionales.

- En el proceso de la investigación se deben definir las competencias, tanto genéricas como específicas, que deben desarrollar los profesionales en su contexto real de trabajo, así como las normas de competencias y los perfiles de desempeño correspondientes. 


\section{Fundamentos teóricos de la metodología}

En la figura 1 se observan los componentes que la integran, en su base se encuentran: la identificación de necesidades de aprendizaje y de las competencias, y dentro de esta última las normas de competencias y los perfiles de desempeño. Existen diferentes metodologías para la identificación de las competencias, entre ellas las más utilizados son el análisis ocupacional, funcional y contructivista.

En el análisis contructivista, el objeto de análisis es la actividad-trabajo y en el análisis funcional, el objeto de estudio es la función productiva, se hace énfasis en la certificación de competencias. El análisis ocupacional presenta como objeto de estudio el puesto de trabajo y la tarea a desarrollar es el proceso de recolección, ordenamiento y valoración de la información sobre las ocupaciones, tanto de las características del trabajo que se realiza como los requerimientos que estas necesitan; en su esencia están las siguientes interrogantes ¿qué hace el profesional, para qué lo hace y cómo lo hace? ${ }^{2}$

En la metodología ECLAS se utiliza el análisis ocupacional sin dejar de considerar e integrar aspectos relacionados con el análisis funcional y contructivista (figura 1).

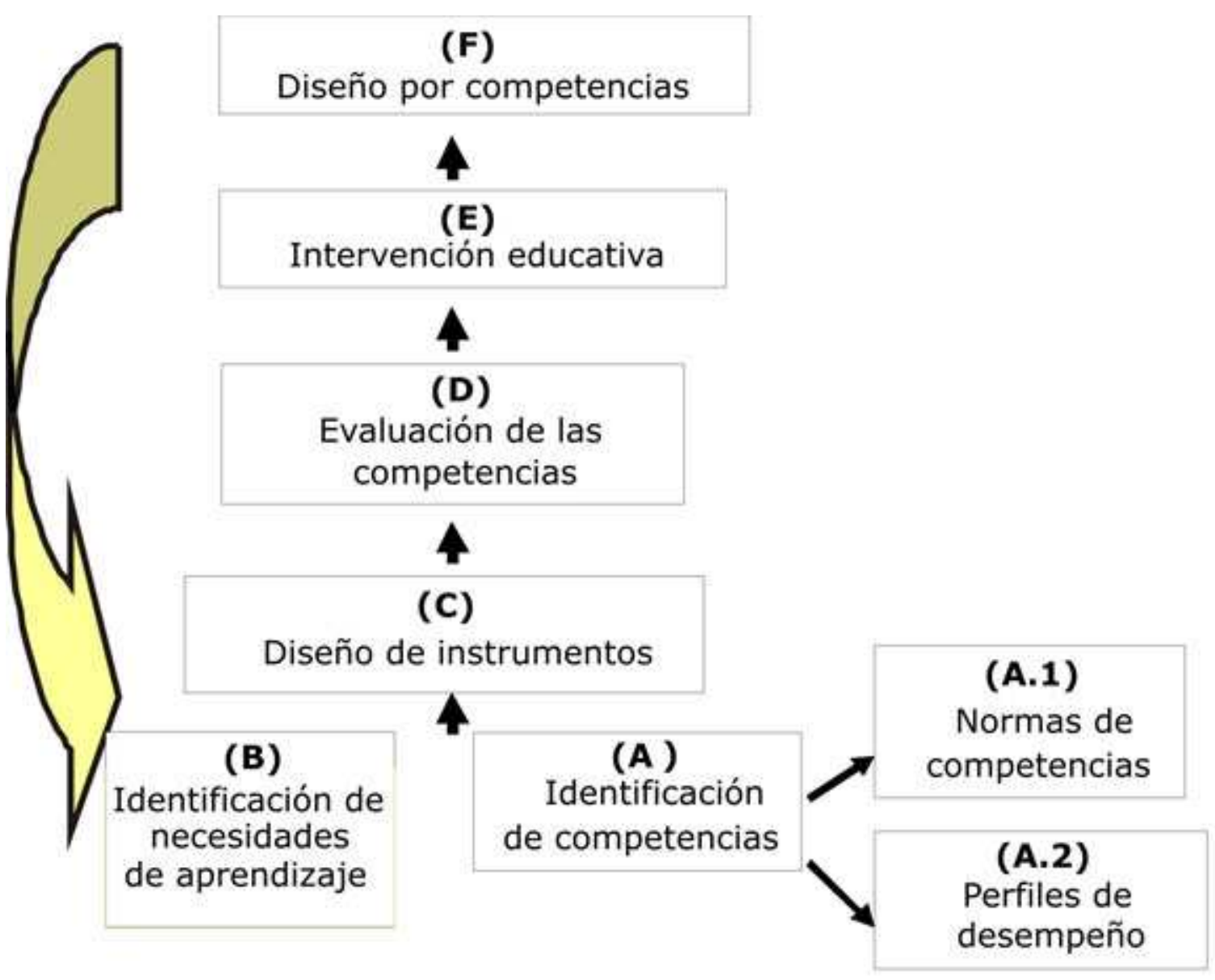

Fig. 1. Metodología ECLAS.

En la literatura se describen fundamentalmente dos metodologías para la realización del análisis ocupacional: a). Desarrollo sistemático e institucional de un currículum (SCID) y b). Desarrollando un currículo (DACUM). ${ }^{10}$ Esta última se utiliza 
para el análisis cualitativo del trabajo, que sigue la lógica de la determinación de la ocupación y se sustenta en los siguientes principios: ${ }^{2}$

- Los trabajadores expertos pueden describir su trabajo más apropiadamente que ningún otro.

- Una forma efectiva de definir una ocupación consiste en describir las tareas que los trabajadores expertos desarrollan.

- Todas las tareas para ser desarrolladas adecuadamente demandan la aplicación de conocimientos, conductas y habilidades, así como la utilización de diferentes equipos y herramientas.

La metodología DACUM ${ }^{10}$ utiliza estos criterios para su trabajo con expertos, pero solo en identificar las competencias, no aplica técnicas de comprobación práctica, por lo que se queda con el criterio subjetivo de los expertos, tampoco realiza intervenciones.

En la metodología ECLAS se asumen los criterios de la metodología DACUM ${ }^{10}$ para el trabajo con los expertos, pero además se deben identificar las necesidades de aprendizaje, diseñar y aplicar instrumentos a partir de las competencias identificadas, las normas y sus perfiles de desempeño y de acuerdo con los resultados obtenidos realizar una intervención educativa en cualquiera de las modalidades de superación profesional del posgrado, se debe realizar además la retroalimentación para iniciar el ciclo nuevamente.

Como se observa en la figura 1 , existen componentes en su base que son: la identificación de las competencias con sus correspondientes normas y perfiles de desempeño y de las necesidades de aprendizaje en los profesionales. Para el trabajo con estos componentes se debe hacer búsqueda bibliográfica con el propósito de indagar en los documentos normativos y metodológicos establecidos por el Viceministerio de Docencia e Investigaciones y la Comisión Nacional de Evaluación de la Competencia y el Desempeño Profesional del Sistema Nacional de Salud.

\section{Identificación de las competencias (A)}

Método o proceso que se sigue para establecer, a partir de una actividad de trabajo, las competencias que se ponen en juego con el fin de desempeñar tal actividad, satisfactoriamente.

\section{Normas de competencias (A.1)}

Es la formalización de una competencia, a través del establecimiento de estándares que la convierten en un referente válido para un determinado colectivo. Conjunto de elementos de competencia, resultante de la negociación entre las diversas partes involucradas en la definición funcional y reglamentación de las condiciones de trabajo de una ocupación o profesión. Describe los conocimientos, las habilidades y operaciones que un individuo debe ser capaz de desempeñar y aplicar en distintas situaciones de trabajo. ${ }^{11}$

\section{Perfiles de desempeño (A.2)}

Se refiere a los aspectos esenciales de una competencia, expresa las características de los resultados y se relaciona con el alcance descrito en el elemento de 
competencia como base para evaluar si un trabajador es o no aún competente. Describe además los requisitos de calidad para el resultado obtenido en el desempeño laboral, que permite establecer si el trabajador alcanza, o no, el resultado indicado en el elemento de competencia. Sustenta la elaboración de material de evaluación y permite precisar acerca de lo que se hace y la calidad con que fue realizado.

Para identificar las competencias, normas de competencias y perfiles de desempeño se debe realizar el análisis del trabajo a desarrollar por el profesional en el servicio y así determinar los conocimientos, habilidades, destrezas, actitudes, aptitudes y motivaciones a fin de lograr los objetivos que la ocupación persigue, que definen las competencias laborales. Para esto se deben crear grupos de expertos constituidos por profesionales propios de la profesión con experiencia en el servicio objeto de análisis y debe estar representada la asistencia y la docencia.

Los comités de expertos deben tener las siguientes características:

- Profesionales con alguna superación profesional (diplomado) o formación académica (especialidad, maestría u otros) en dependencia de la especialidad que se vaya a analizar.

- Ser jefes de salas, servicios o docentes-asistenciales.

- Tener entre 5 y 10 años de experiencia en el servicio.

En este tipo de técnica se designa un grupo de personas expertas en determinados aspectos, las cuales se reúnen periódicamente con el propósito de profundizar en elementos esenciales a tener en cuenta para el cumplimiento de los objetivos y finalidades para los que fueron convocados; entre las ventajas descritas está que permite agrupar opiniones y puntos de vista complementarios que facilitan el proceso, crear un clima favorable para la discusión e incluir enfoques adicionales relacionados con los trabajadores, especialistas y dirigentes. Son útiles además para explorar conocimientos, experiencias y pueden ser usadas para examinar no solo lo que la gente piensa, sino, cómo piensa y por qué piensa de determinada manera. Esta técnica es apropiada cuando el investigador tiene preguntas abiertas y desea estimular a los participantes a explorar determinados hechos de importancia. ${ }^{12,13}$

Una vez definidas las competencias que debe desarrollar el profesional, la interrogante siguiente sería ¿cómo garantizar que sean esas las competencias y no otras? Para ello se debe consultar con otro grupo de expertos que esté relacionado con el trabajo de la profesión en cuestión y que posea requisitos de preparación académica y científica y profesionales que tengan que cumplir las competencias identificadas.

El comité de expertos debe efectuar varias rondas de trabajo y el investigador principal actuar como moderador en las sesiones de análisis y discusión, teniendo como premisa las funciones asistenciales que desarrolla el profesional. Deben analizarse aspectos que son determinantes en la identificación de las competencias laborales, entre ellos se encuentran:

- Capacidades para la transferencia y generalización de la información.

- Trabajo en equipo, saber comunicarse y colaborar con sus miembros. 
- Utilización de las tecnologías de información y comunicación.

- Dominio de un idioma extranjero.

- Desarrollo profesional continuado y ponerlo en práctica en su vida diaria.

- Habilidades para definir, identificar y solucionar problemas.

- Pensamiento crítico.

- Compromiso activo para desarrollar con éxito sus objetivos de trabajo.

- Aptitudes de autodesarrollo, autonomía y autoevaluación.

- Cultura general y política.

- Conocimiento general de aspectos sociales, económicos, tecnológicos y ambientales vinculados al ejercicio de la profesión.

- Respeto al trabajo propio y el de los demás.

- Honestidad, responsabilidad y solidaridad.

- Compromiso social, político e institucional.

Posteriormente después de analizados estos aspectos se realizará la propuesta de las competencias genéricas y específicas a desarrollar por los profesionales en el servicio en cuestión. En las dos últimas sesiones de trabajo se llega al consenso final de la propuesta de las competencias laborales. Finalmente se trabaja en un instrumento donde aparezcan las competencias propuestas, el cual se caracteriza por una escala valorativa del uno al cuatro, el número mayor se corresponde con el nivel más alto que le da cada experto en particular a la competencia en cuestión.

Para constatar la pertinencia de las competencias específicas identificadas por el comité de expertos y conocer los criterios de otros profesionales y miembros del equipo de trabajo que interactúan con este personal, se realizaron estudios de validez de contenido con el instrumento diseñado por el comité de expertos, para lo cual se utilizó el criterio de:

- Otro grupo de expertos conformado por médicos, que cumplieran la condición de ser especialistas del servicio en cuestión, así como los jefes de los servicios de las instituciones seleccionadas.

- Otros profesionales donde el tiempo de graduados esté comprendido entre 1 y 5 años y encontrarse activos en su función asistencial... ${ }^{14}$

Para el grupo de expertos la técnica utilizada fue la entrevista grupal. Esta ha evolucionado hacia un tipo de encuentro, donde una persona, típicamente identificada como profesional, recaba información de otras, y desde el punto de vista de la investigación científica se torna en un método que permite la obtención de datos. El uso de este tipo de entrevista es apropiado cuando se abordan temas de cierta complejidad, que requiere de la explicación detallada de algunos aspectos, en beneficio de una comprensión adecuada, ${ }^{15}$ como es el caso de la que se aplica en esta investigación. Con este segundo grupo de expertos el investigador principal también actúa como facilitador y explica la importancia de sus criterios en relación 
con las competencias que fueron propuestas por el comité de expertos de la profesión relacionados con la especialidad en cuestión, después de ofrecerles una información detallada, se les entrega el instrumento donde aparecen las competencias específicas, las que fueron ponderadas a partir de la escala valorativa explicada en el comité de expertos.

\section{Identificación de las necesidades de aprendizaje (INA) (B)}

Se define como la desviación real entre el desempeño práctico y el que el sistema tiene previsto en esa función o puesto de trabajo, siempre que esa diferencia obedezca a falta de conocimientos, preparación o entrenamiento. ${ }^{16}$ Constituye una herramienta que debe aplicarse de manera participativa según los contextos laborales. Comprende la exploración de las necesidades sentidas y de los conocimientos teóricos y prácticos, se debe realizar sobre la base de la identificación previa de las competencias laborales. La INA constituye una trascendental herramienta de la educación permanente, la cual debe aplicarse con un enfoque sistémico y participativo.

El carácter dinámico e histórico de las necesidades de aprendizaje no es un acto aislado y discontinuo, por el contrario, es constante, periódico y permanente, es un paso previo a la definición del contenido, los métodos y la organización de cualquier tipo de proceso educativo en el ámbito laboral. Entre las ventajas se describe que:

- Proporciona elementos necesarios para elaborar diferentes modalidades de superación profesional requeridos para cumplir las metas y políticas de salud.

- Suministra elementos complementarios sobre dificultades y problemas ajenos al de los profesionales.

- Erradica gastos innecesarios, centrando los esfuerzos en lo efectivo.

- Induce a una aceptación general de la capacitación, al lograr un programa de trabajo que todos los factores aceptan en respuesta a los problemas que confrontan.

- Proporciona datos y elementos que permiten un seguimiento y comparación del salto cualitativo en cada lugar concreto.

Para identificar las necesidades de aprendizaje existen varios instrumentos, uno de los más usados es la encuesta, su diseño deberá estructurarse con preguntas abiertas y cerradas para la obtención de información. Este aspecto es de vital importancia si se parte del principio de la participación que es sentirse parte de, lo que además está sustentado en algunos antecedentes y definiciones de empoderamiento. Según Carnota Lauzán ${ }^{17}$ el enfoque participativo, sobre todo en cuanto al trabajo con equipos autodirigidos, logrará dotar al personal de autoridad y conocimientos y en función de ello, otorgarles poder de decisión sobre la actividad que realizan.

Son ejemplos de técnicas e instrumentos a utilizar para la INA: entrevistas, cuestionarios, evaluación del desempeño, reuniones de grupo, observación, encuestas, lista de verificación, tormenta de ideas, conversación informal y simulación, entre otras.

\section{Diseño de instrumentos evaluativos (C)}


Los instrumentos se diseñan en dependencia de las competencias, normas de competencias y perfiles de desempeño identificados a desarrollar por los profesionales y deben cumplir con las características de transparencia, objetividad, confiabilidad y validez. ${ }^{17}$

- La transparencia se refiere al hecho de que todos los actores (candidatos, evaluadores, directivos y formadores) conozcan lo que se debe demostrar.

- La objetividad implica que los juicios se realicen sobre la evidencia que realiza el individuo y no sobre las creencias o expectativas que el evaluador tiene sobre él.

- La confiabilidad significa que debe obtenerse evidencia similar cuando el instrumento lo aplican diferentes evaluadores o cuando el instrumento aplicado en diferentes circunstancias arroja resultados consistentes a través del tiempo.

- La validez está asociada con los instrumentos que se utilizan para recoger la evidencia, los cuales deben corresponder a las competencias identificadas al respecto.

En el diseño de los instrumentos se deben considerar aspectos de la teoría y la práctica, lo que permite obtener información relacionada con conocimientos, habilidades, dominio de las tecnologías, calidad de la atención y aspectos que consideren las actitudes ante situaciones o problemas que pudieran presentarse en el servicio; es aconsejable la combinación de instrumentos para poder realizar una valoración general de las acciones que desarrollan los profesionales. Se recomienda el uso de instrumentos evaluativos escritos y la utilización de preguntas tipo test; la observación en los servicios para la verificación en la práctica y finalmente para poder lograr una evaluación integral, utilizar la auditoria como vía para determinar la calidad del servicio prestado.

En general, entre los documentos de obligada consulta para el diseño de instrumentos evaluativos, están el Anteproyecto de Evaluación de la Competencia y el Desempeño Profesional en el Sistema Nacional de Salud ${ }^{18}$ y la Indicación Metodológica 7/98 la cual norma el procedimiento para la confección de instrumentos evaluativos escritos. ${ }^{19}$ La impartición de talleres con la participación del comité de expertos es una tarea a desarrollar para trabajar en la selección de los temas y procedimientos a evaluar.

Instrumentos evaluativos escritos. Dentro de este tipo de instrumento, los exámenes escritos son mundialmente los de mayor uso, los más económicos y los de mayor flexibilidad técnica; deben reunir particularidades generales, las cuales estarán dadas por las características del contenido, su importancia y utilidad, relación con los objetivos, tener validez y confiabilidad, objetivo y factible de realizar, complejo, pero lo más breve y preciso posible, sin detrimento de la claridad en su redacción. ${ }^{20}$

Para el diseño de este instrumento escrito se deben analizar las competencias laborales que caracterizan a los profesionales en el servicio y a partir de estas se realizará la selección de los temas objetos de comprobación para la exploración del nivel de actualización cientificotécnica. Para esta selección se debe tener en cuenta: los principales problemas de salud de la población del territorio, la identificación de necesidades de aprendizaje detectadas en inspecciones realizadas y los resultados de la encuesta.

Se elabora la tabla de especificaciones de contenido (figura 2), que incluye los contenidos y los niveles de asimilación que se utilizaran en el proceso de la 
evaluación. Es una tabla de doble entrada en uno de cuyos márgenes se ubica un listado de temas a evaluar y en la otra los niveles de asimilación. Su abordaje se basa en que las tareas clínicas deben ser examinadas no de forma aislada, sino en correspondencia con problemas concretos de salud.

\begin{tabular}{|l|l|l|l|l|l|}
\hline \multirow{2}{*}{ No. } & \multirow{2}{*}{ Temáticas } & \multicolumn{3}{|c|}{ Niveles de Asimilación } & \multirow{2}{*}{ Total } \\
\cline { 3 - 5 } & & Reproducción & $\begin{array}{c}\text { Aplicación de } \\
\text { técnicas y } \\
\text { procedimientos }\end{array}$ & $\begin{array}{c}\text { Identificación y } \\
\text { solución de } \\
\text { problemas }\end{array}$ & \\
\hline & & & & & \\
\hline & & & & & \\
\hline & & & & & \\
\hline
\end{tabular}

Fig. 2. Tabla de especificaciones de contenido.

El instrumento evaluativo debe ser diseñado para examinar los conocimientos que sean esenciales o claves para la solución satisfactoria del problema, o aquellos que se espera que los evaluados sean capaces de realizar de forma independiente. ${ }^{21,22}$

Para que el instrumento que se diseñe esté acorde con lo expuesto con anterioridad se debe realizar el análisis de aspectos que lo caracterizan:

- Definición del dominio cognitivo de los temas a evaluar.

- Correlación de preguntas con los temas a evaluar.

- Distribución porcentual de los formatos y preguntas por temas.

- Puntaje por preguntas.

Dentro de las preguntas tipo test que se utilizan para la confección del instrumento se encuentran las de: verdadero-falso, selección múltiple complemento simple, selección múltiple complemento agrupado, enlace o apareamiento y ensayo de respuestas cortas, estos formatos de preguntas tienen ventajas y desventajas en su aplicación. ${ }^{21}$

Para la determinación de la fiabilidad del instrumento, es recomendable utilizar el "Coeficiente $\alpha$ de Crombach" porque: ${ }^{23,24}$

- Es el más utilizado en estudios contemporáneos de calidad de instrumentos evaluativos, lo que permite comparar resultados con los que se reportan en la literatura mundial.

- Proporciona la medida de la consistencia interna del examen en cuestión.

- Puede calcularse aún en el caso de que se empleen preguntas de diferentes formatos.

El análisis de fiabilidad se realiza sobre la base de los cálculos del mencionado coeficiente para el examen considerado como un todo y para cada una de sus temáticas.

\section{Guía de observación}


Instrumento utilizado para la verificación de las habilidades prácticas de los profesionales en el servicio, se utiliza la observación directa no participante y estructurada, ${ }^{25}$ los observadores se ponen en contacto directo con el fenómeno a investigar, se obtiene la información desde afuera y se realiza con la aplicación de la guía, el observador tiene la posibilidad de marcar cada vez que observa el cumplimiento o no de la habilidad descrita.

Se confecciona una guía de habilidades a desarrollar, la cual se entrega a cada observador. Esta evaluación, a partir de la obtención de la información de lo que realmente realiza el profesional en el servicio, es un juicio que define si este es competente o aún no competente, se centra en demostrar si existen las habilidades requeridas para un desempeño adecuado.

Existen diferentes formas para la evaluación de las competencias laborales, en la metodología ECLAS se tienen en cuenta tres aspectos, los cuales podrán demostrar que el profesional puede desempeñar la actividad descrita, según los diferentes criterios establecidos, las formas son: registros en el desempeño del trabajo, evidencia histórica y actualización cientificotécnica. ${ }^{2}$

Registros en el desempeño del trabajo. Se refieren a todas aquellas actividades que el profesional realiza cotidianamente durante su trabajo. En este desempeño puede encontrarse los diferentes modos de actuación del profesional ante situaciones que se le presentan en su contexto laboral, lo que constituye una forma de obtener evidencia y sus resultados son considerados imprescindibles dentro de este enfoque. De esta forma la evaluación involucrará la observación de las actividades en el momento en que ocurren y el análisis de los resultados.

La evidencia histórica. Se refiere a todas aquellas actividades que el profesional ha desempeñado en su trabajo y fuera del marco laboral. Se refiere a aspectos de investigación científica, publicación de artículos, participación en eventos, participación en alguna modalidad de superación profesional (cursos, entrenamientos, diplomados) o formación académica (especialidades, maestría o doctorado) y participación en la docencia, entre otras.

La actualización cientificotécnica. Tendrá sus bases sobre la aplicación de instrumentos escritos, los cuales contarán con preguntas de tipo test de diferentes tipos.

El procedimiento de la evaluación estará constituido por tres etapas: elaboración del plan de evaluación, integración de las evaluaciones y la emisión del juicio. ${ }^{2}$

La elaboración del plan de evaluación es la acción mediante la cual se explicita la estrategia de la evaluación, que será comunicada y discutida con los profesionales. La integración de las evaluaciones es la recopilación de evidencias o resultados de las diferentes evaluaciones realizadas, que demuestran las competencias del profesional y la emisión del juicio de competencias es el resultado de la comparación de las evidencias recopiladas en la integración de las evaluaciones realizadas contra los criterios de competencias, su resultado puede ser: competente o todavía no competente.

Para hacer más explícito el proceso de evaluación, se construyó un algoritmo que se representa en la figura 3 . En este se pone de manifiesto el carácter cíclico que posee la evaluación, pues es el componente de la metodología donde se materializa la retroalimentación. 


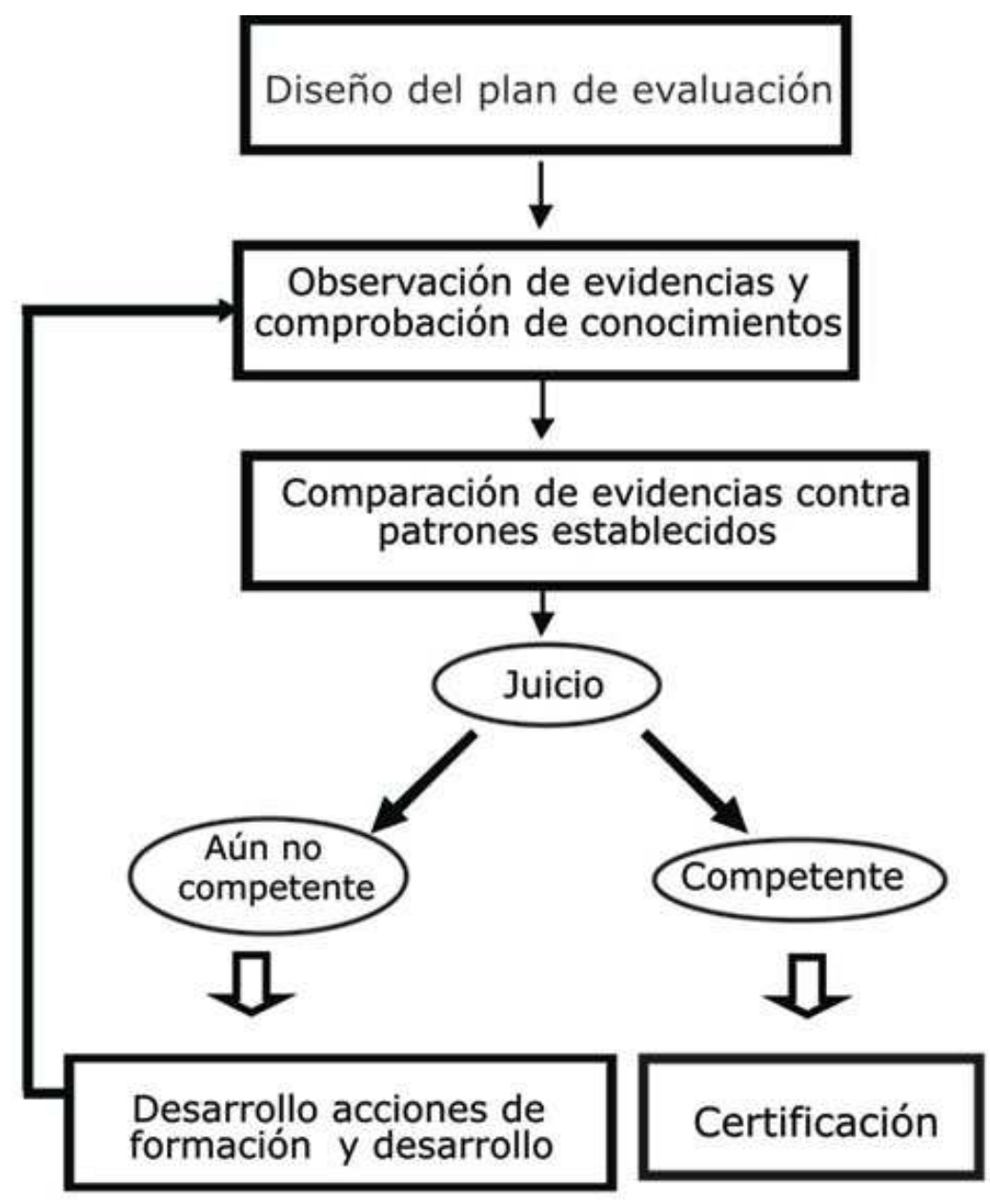

Fig. 3. Momentos de la evaluación de las competencias.

Los observadores serán los integrantes del comité de expertos y tendrán la característica de tener suficientes conocimientos del servicio y con las acciones y procedimientos a realizar por los profesionales. La observación se inicia a las 8 a.m. y culmina al finalizar el profesional todos los procederes establecidos en la guía de observación, en los casos de que no fuera posible, se concluye al día siguiente. Al finalizar la observación se le da a conocer al profesional evaluado los resultados, sugerencias y recomendaciones pertinentes para cada caso.

Para la selección de los profesionales a observar en los servicios, se debe utilizar un diseño no probabilístico, según criterio razonado de autoridad. Esta es, sin duda, la estrategia de muestreo más común y consiste en orientar la selección de acuerdo con estrictos criterios de costo y rapidez.

Para la selección de los servicios, se consideraran criterios imprescindibles relacionados con la estructura física del servicio, equipamiento técnico conveniente, disponibilidad de ropa estéril en caso necesario y disposición del personal profesional.

Para el proceso de observación se estructura una clave de evaluación donde en su diseño aparecen los pasos a seguir, las diferentes situaciones en la que se pudiera encontrar el observador y la calificación que debiera otorgar, todo ello fundamentado en aspectos que se encuentran en el modelo de observación. 
La calificación de la técnica o procedimiento asistencial se realiza cualitativamente. En la confección de la guía de observación se consideran aspectos invalidantes, los cuales el profesional no puede dejar de realizar y como consiguiente tendría un peso importante en la calificación a obtener.

\section{Auditoria}

La auditoria es otro instrumento diseñado tomando como referencia aspectos de la auditoria médica referidos en el libro "Tecnologías Gerenciales" de Carnota Lauzán ${ }^{17}$ donde se dice que la conveniencia de efectuar una auditoria está originada por diversas causas:

- Resultados de la interacción con el equipo de trabajo, o con los pacientes, donde se determinaron problemas relacionados con el servicio.

- Diferencias en el desempeño de profesionales de un servicio con respecto a otros, en otras instituciones.

- Quejas de pacientes, familiares y la comunidad asociadas al servicio.

Además plantea, que el aplicar una auditoria se detectan problemas de mal funcionamiento que existen, que lo conoce o no la institución, que ha sido detectado por ella misma o por terceros, o que se sospecha que se puede estar presentando; en cualquiera de los casos se necesita una confirmación o no, determinar sus causas y actuar posteriormente.

Las auditorias tienen un carácter retrospectivo y se orientan a problemas concretos acerca de los cuales se revisan las actuaciones y decisiones de los profesionales en su quehacer diario. Su base de trabajo lo constituyen las historias clínicas. Es una herramienta que puede ser utilizada por todos los profesionales de la salud para mejorar la atención a los pacientes. El espectro de aspectos susceptibles de auditar es amplio y muchas áreas de trabajo que no se evaluaban han sido sometidas a evaluación. A menudo esto ha servido para demostrar la falta de conocimiento acerca de un área en particular, que a su vez ha estimulado una mayor investigación.

Siempre han existido formas de auditar en los centros asistenciales, pero recién en los últimos años se han comenzado a realizar según el concepto formal, que implica la participación de cada vez más profesionales y especialistas. El alto grado de participación en ellas y la gran cantidad de evidencias que surgen del anecdotario personal demuestran que muchas de las auditorias son realizadas por profesionales que no conocen a fondo las metodologías de investigación, o que no tienen experiencia previa en la investigación y las cuestiones éticas relacionadas. ${ }^{26}$

La auditoria está dirigida a identificar la calidad de las actividades de los profesionales en el servicio, que permite explorar aspectos relacionados con la organización en el servicio y la aplicación de los principales lineamientos para la confección de las historias clínicas, lo que redundará en mejores resultados en la atención.

Para lograr la efectividad en la aplicación de auditorias, se mantuvo el trabajo con el comité de expertos donde los expertos poseen un nivel técnico científico igual o superior al de los profesionales objetos de la evaluación y están familiarizados con los procedimientos y las actividades a desarrollar por los profesionales en el servicio. La auditoria se realiza al concluir la observación en el servicio. 


\section{Intervención educativa (E)}

A partir de los resultados obtenidos en la aplicación de los instrumentos evaluativos, se debe realizar una intervención educativa en cualquiera de las figuras de superación profesional. Para el diseño curricular del programa es necesario partir de una reflexión sobre los cambios que se espera que ocurran en los profesionales a través del aprendizaje. Habría que preguntarse entonces: ¿qué sabía antes del programa y qué sabrá después?, ¿qué podía hacer antes del programa con lo que sabía y qué podrá hacer después?, ¿en qué sentido el programa va a ayudar al desarrollo de estos profesionales?

El diseño curricular de planes y programas reconoce diversas fuentes culturales y socioocupacionales hasta llegar a una síntesis que serían los aprendizajes necesarios. Las planificaciones analíticas han cedido paso a modelos holísticos y sistémicos a través de los cuales se procura atender la complejidad del diseño. El diseño por competencias presenta varias diferencias con la formación convencional o tradicional, pero no parece haber duda de que una es la diferencia más clara, definitiva y obvia: La Formación Basada en Competencias que tiene como referente una competencia y eso obliga a que su diseño curricular se ordene desde el comienzo en torno a un desempeño, no podría partir, como sucede a menudo con programas de corte academicista, de los contenidos de una disciplina ni de lo que un grupo de profesores considera que las personas deberían aprender. ${ }^{2}$

Para el diseño del programa se recomienda realizar sesiones de trabajo metodológico con el comité de expertos que participa en la investigación para la selección de los temas y prácticas que estarán presentes en el programa, haciendo énfasis en las principales insuficiencias y deficiencias encontradas en las evaluaciones realizadas a los profesionales y las competencias identificadas.

\section{CONSIDERACIONES FINALES}

1. La integración de cada uno de los componentes de la metodología ECLAS para la evaluación de las competencias laborales y su interacción dinámica, donde cualquier cambio en uno afectará a los demás, le da el carácter sistémico.

2. La base de la metodología para la evaluación de las competencias laborales la constituye la identificación de las competencias, normas de competencias y perfiles de desempeño, y de las necesidades de aprendizaje, todas se interrelacionan entre sí. Para una mejor identificación de necesidades de aprendizaje previamente se deben identificar las competencias, sus normas y perfiles.

\section{REFERENCIAS BIBLIOGRÁFICAS}

1. Gallart AM, Jacinto C. Competencias laborales: tema clave en la articulación educación. Madrid: OEI;1997.

2. Irigoin $M$, Vargas $F$. Competencia laboral: manual de conceptos, métodos y aplicaciones en el sector salud. Montevideo: Cinterfor/OIT;2002.

3. Metodología para la ordenación de la formación profesional ocupacional. Subdirección general de gestión de formación ocupacional. Madrid: INEM;1995. 
4. Urbina Laza O. Evaluación de las Competencias en Enfermería. La Habana: ENSAP;2002.

5. Gimeno Sacristán J. La evaluación de la enseñanza. En: Pérez Gómez Al, Mac Donald B, editores. La evaluación: su teoría y su práctica. Caracas: Cooperativa Laboratorio Educativo;1993.p.338.

6. Vargas F. La formación basada en competencias: instrumento para la empleabilidad. Montevideo: Cinterfor/OIT;1999.

7. Sabino Carlos A. El proceso de investigación [sitio en Internet]. [citado 23 de febrero de 2007]. Disponible en:

http://www.latinlog.net/blog/wp-content/uploads/2009/09/metodologia.pdf

8. Washington Puig R, Más García M. El enfoque sistémico en el contenido de la enseñanza. Rev Cubana Educ Med Sup. 2003;17(2).

9. Urbina Laza O. Metodología para la evaluación de las competencias laborales de los profesionales de enfermería que laboran en los servicios de neonatología [tesis]. La Habana: Escuela Nacional de Salud Pública;2007.

10. Torres Esperón M. Metodología para definir funciones profesionales. Rev Cubana Salud Pública [serie en Internet]. 2008 Dic [citado 2010 Ene 22];34(4):

Disponible en: http://scielo.sld.cu/scielo.php?script=sci arttext\&pid=S0864$\underline{34662008000400017 \& \operatorname{lng}=\mathrm{es}}$

11. Avance Conceptual y Metodológico de la Formación Profesional en el Campo de la Diversidad en el Trabajo y de la Certificación Profesional. Brasil: MTE;2004.

12. Callejo J. Articulación de perspectivas metodológicas: capacidades del grupo de discusión para una sociedad reflexiva. Rev Papers. 1998;56:31-55.

13. Callejo Gallego J. Observación, entrevista y grupo de discusión: el silencio de tres prácticas de investigación. Rev Esp. 2002;76(5).

14. Torres Esperón M. Funciones de Enfermería según niveles de formación. Propuesta para el Sistema de Salud Cubano [tesis]. La Habana: Escuela Nacional de Salud Pública;2006.

15. León Nacedo I, Abreu Guerra E. Metodología de la Investigación Pedagógica y Psicológica. 2da. parte. La Habana: Editorial Pueblo y Educación;1989.

16. Hatim R. Identificación de Necesidades de Aprendizaje [monografía en CDROM]. La Habana: ENSAP;2004.

17. Carnota Lauzán O. Tecnologías Gerenciales. Una oportunidad para los sistemas de salud. La Habana: ENSAP;2005.

18. Vargas F. Competencias claves y aprendizaje permanente. Montevideo: Cinterfor/OIT;2004.

19. Anteproyecto de Evaluación de la Competencia y el Desempeño Profesional en el Sistema Nacional de Salud. La Habana: CENAPEM;2000. 
20. Indicación Metodológica 7/98 del Viceministerio de Docencia e Investigaciones. La Habana: MINSAP; 1998.

21. Soler Cárdenas SF, Salas Perea RS. Calidad de los instrumentos evaluativos. La Habana: MINSAP;1997. [ Carpeta No.4].

22. García Hernández, Salas Perea RS, Soler Cárdenas SF. Consideraciones para planear un instrumento de evaluación. La Habana: MINSAP;1997. [Carpeta No.3].

23. García Hernández, Salas Perea RS. La construcción de instrumentos evaluativos escritos. Elementos para mejorar su calidad. La Habana: MINSAP;1997. [Carpeta No.2].

24. Soler Cárdenas S, Salas Perea RS. La calidad de los instrumentos evaluativos. La Habana: CENAPEM; 1997.

25. Bacallao J, Alerm A, Artiles L. Texto básico de metodología de la investigación educacional [monografía en CD-ROM]. La Habana: ENSAP;2004.

26. Agranatti P. Auditoria y bioética[sitio en Internet]. [citado 10 de abril del 2006]. Disponible en:

http://www.odontomarketing.com/numeros\%20anteriores/ART 56 AGOSTO 2002. $\underline{\text { htm }}$

* Función asistencial: está encaminada a apoyar al individuo en la conservación y el incremento de su salud. Responde al cuidado de las personas enfermas y sanas, es decir, ayudarlas a mantener su salud o recuperar las funciones y capacidades deterioradas por un proceso patológico. Para ello la función asistencial se desarrolla tomando los 3 niveles de atención de salud.

Recibido: 18 de diciembre de 2008.

Aprobado: 20 de mayo de 2009.

Omayda Urbina Laza. Escuela Nacional de Salud Pública. Calle Línea esq. a I. El Vedado 10400. La Habana, Cuba.

E-mail: omayda@ensap.sld.cu 